\title{
Non-linear Elastic Behaviour of Unbound Aggregates in FEM representing Railway Transition Zones
}

\author{
J.N. Varandas ${ }^{1}$, A. Paixão ${ }^{2}$, E. Fortunato ${ }^{2}$, P. Hölscher ${ }^{3}$ and R. Calçada $^{4}$ \\ ${ }^{1}$ UNIC, Department of Civil Engineering, Nova University of Lisbon, Portugal \\ ${ }^{2}$ Transportation Department, National Laboratory for Civil Engineering \\ Lisbon, Portugal \\ ${ }^{3}$ Geo-Engineering Department, Deltares, Delft, The Netherlands \\ ${ }^{4}$ Department of Civil Engineering, Faculty of Engineering \\ University of Porto, Portugal
}

\begin{abstract}
The stress dependent behaviour of the unbound granular materials that carry the track, and the voids that may occur between the base of the sleepers and the ballast, are the important factors for non-linear track behaviour. On transition zones, where the track stiffness may rapidly vary and often differential settlements take place between the stiff structure and the approach section of the track, these aspects are of particular relevance. Despite the non-linear constitutive behaviour of unbound aggregates, it is still common practice to consider the linear elastic behaviour for these materials in the design of transportation infrastructure. The present study focuses on the implementation of these non-linear aspects in a finite element algorithm for the dynamic simulation of railway tracks. Linear and nonlinear constitutive laws are used to reproduce the behaviour of unbound aggregates that comprise the trackbed. Stress and deformation results are presented and discussed for both linear and non-linear scenarios. The results show that the consideration of these non-linear aspects is relevant for the modelling of railway transitions.
\end{abstract}

Keywords: railways, non-linear, constitutive models, unbound aggregates, transition zones.

\section{Introduction}

Transition zones in railway tracks are built to mitigate the impact caused by structural discontinuities along the track. These discontinuities may be bridge approaches, passages over culverts, at road and rail crossings, ends of tunnels, or passages from slab tracks to ballasted tracks. Such places frequently show accelerated track geometry degradation and increased wear and tear on track and vehicle components. This 